\title{
MODERNIZAÇÃO DA INSPEÇÃO E AVALIAÇÃO DE ESTRUTURAS: AS CONTRIBUIÇÕES DE VANTS E ROBÔS NA ÁREA DE PATOLOGIA DAS CONSTRUÇÕES
}

\author{
SANTOS, RODRIGO FELIPE \\ Doutorando em Engenharia da Construção \\ Universidade Federal de Viçosa \\ Minas Gerais; Brasil \\ rodrigo.felipe@ufv.br
}

\author{
SILVA, REGINALDO CARNEIRO \\ Doutor em Engenharia de Estruturas \\ Universidade Federal de Viçosa \\ Minas Gerais; Brasil \\ recsilva@ufv.br \\ RIBEIRO, JOSÉ CARLOS LOPES \\ Doutor em Engenharia de Estruturas \\ Universidade Federal de Viçosa \\ Minas Gerais; Brasil \\ jcarlos.ribeiro@ufv.br
}

\author{
SANTOS, RUAN RICHELLY \\ Mestrando em Engenharia de Estruturas \\ Universidade Federal de Minas Gerais \\ Minas Gerais; Brasil \\ ruanrichelly@gmail.com
}

\author{
MENDES, BEATRYZ CARDOSO \\ Doutoranda em Engenharia da Construção \\ Universidade Federal de Viçosa \\ Minas Gerais; Brasil \\ beacm03@gmail.com
}

\section{RESUMO}

No que tange o comportamento das estruturas ao longo de sua vida útil, são vários os problemas que podem acometer as construções, desde defeitos estéticos até sua ruína nos casos mais graves. Dado o potencial risco, as construções devem ser inspecionadas periodicamente no intuito de descobrir precocemente as manifestações patológicas e, a partir do diagnóstico, serem propostas as devidas intervenções. Com o contínuo avanço da ciência, tem-se buscado utilizar a tecnologia para aprimorar o monitoramento, inspeção e avaliação das estruturas. Nesse sentido, este trabalho tem por objetivo mostrar as potencialidades do emprego de veículos aéreos não tripulados - VANTs (também conhecidos como drones) e robôs na construção civil, tornando a coleta de dados mais viável e até mesmo automatizada. Por meio de uma revisão bibliográfica da aplicação desses equipamentos, fica evidente que este tema é promissor, com muitas pesquisas ainda em andamento. O emprego de drones e robôs permite eliminar a subjetividade e erros humanos durante a inspeção, além de possibilitar o alcance a locais de difícil acesso ou de grande risco para as pessoas, como áreas de acidente, por exemplo. Diferentes dados podem ser levantados em função dos equipamentos instalados nos veículos remotos, como câmeras, GPS e lasers de escaneamento. Após o tratamento das informações obtidas, que pode ser tanto manual quanto automatizado, obtém-se um quadro do estado da estrutura avaliada. Assim, percebe-se que a adoção de drones e robôs tem muito o que acrescentar e colaborar para a área de Patologia das Construções.

Palavras-chave: Patologia das Construções, Inspeção e Monitoramento, tecnologia.

\section{ABSTRACT}

Several problems can affect the structural behavior of constructions during their life cycle, from aesthetic defects to the collapse in more severe cases. Their condition needs to be monitored and inspected in order to ensure their safety and functionality, assessing early pathological manifestations and allowing appropriate interventions. Nowadays, new technologies offer promising solution to the monitoring, inspection and condition assessment of structures. In this sense, this work aimed to display an overview of the potentialities of unmanned aerial vehicles (UAVs), unmanned ground vehicles (UGVs) and unmanned marine vehicles (UMVs), which can provide rapid and automated inspection and monitoring for civil infrastructure condition assessment. Thus, through a literature review of the application of these devices, it is possible to demonstrate the promising aspect of these solutions. Their application can minimize the subjectivity and mistakes that can occur from a visual inspection performed by trained inspectors. Moreover, this technology allows the condition assessment in dangerous or hard to access areas. Different types of data can be collect depending on the type of devices installed on these vehicles, such as cameras, GPS and scanning lasers. A viewpoint of the structural condition is obtained after information processing, which can be either manual or automated. Therefore, it is possible to conclude that the adoption of these new inspection and monitoring technologies have much to contribute to the field of Building Pathology.

Keywords: Building Pathology, Inspection and Monitoring, Technology. 


\section{INTRODUÇÃO}

As edificações são construídas para diversos fins, devendo atender a sociedade de forma satisfatória ao longo de suas vidas úteis. E para que essa premissa seja atendida, elas precisam manter boas condições de uso. No entanto, por razões internas e externas, diferentes manifestações patológicas podem surgir, afetando a integridade e funcionalidade da estrutura. Assim, é essencial que haja um monitoramento frequente para se evitar ou minimizar os efeitos danosos dessas manifestações.

A preocupação com a manutenção das construções já deve começar na etapa de concepção do projeto. Deve-se buscar soluções que minimizem a degradação dos materiais e facilitem o monitoramento de pontos críticos, mais propensos ao desenvolvimento de manifestações patológicas de qualquer natureza. Já na etapa de execução deve-se garantir o cumprimento do projeto e evitar quaisquer tipos de erros inerentes a esta etapa, sobretudo falhas humanas. E por fim, com a obra concluída, programas de manutenção periódica e preventiva devem ser adotados a fim de identificar problemas na fase inicial ou até mesmo antes que surjam. Em função das informações obtidas nas inspeções periódicas, podem advir inspeções condicionadas, mais direcionadas (SOUZA; RIPPER, 2009).

Nesse contexto, diferentes técnicas e ferramentas são usadas na inspeção e monitoramento das estruturas. Nas vistorias iniciais conta-se sobretudo com os cinco sentidos do avaliador. Conta-se também com certos instrumentos que ampliam esses sentidos, como binóculos, martelos de percussão, câmeras fotográficas, fissurômetros, réguas, fios de prumo, entre outros. Conforme os problemas analisados e o local onde ocorrem, apenas essa operação já é suficiente para se chegar a um diagnóstico (LICHTENSTEIN, 1986).

No entanto, alguns problemas são mais complexos, de observação mais trabalhosa e ocorrem em lugares de difícil acesso ou que conferem algum nível de risco. Em tais situações, são requeridas técnicas e instrumentos mais sofisticados, que expandam a capacidade de inspeção e monitoramento das estruturas. Graças ao contínuo desenvolvimento tecnológico, já existem soluções para essas questões, como os veículos controlados remotamente, que podem executar serviços em ar, solo e água, o que os torna altamente abrangentes e eficazes.

O objetivo deste artigo é abordar as soluções mais atuais no monitoramento e inspeção de estruturas, evidenciando suas características e potencialidades.

\section{EMPREGO DA TECNOLOGIA NA INSPEÇÃO E MONITORAMENTO DE ESTRUTURAS}

Para garantir a segurança e funcionalidade das edificações, é crucial que sejam feitas inspeções para identificação de possíveis problemas. As inspeções visuais manuais, ou seja, aquelas feitas por humanos, são a principal prática de avaliação das estruturas. Embora estas inspeções demandem grandes gastos para as agências governamentais, como ocorre nos Estados Unidos, por exemplo, nem sempre elas são suficientes ou possíveis de ocorrerem (KOCH et al., 2015).

As inspeções manuais demonstram algumas limitações sérias: são demoradas; exigem um trabalho intenso dos profissionais; são subjetivas por demandar a interpretação dos sinais observados; e podem ser arriscadas quando efetuadas em locais na iminência de ruir ou que sofreram um grave acidente (HOSKERE et al., 2017).

Uma forma de contornar esses problemas é tornar o processo mais automatizado, o que diminui a intervenção humana, os custos e melhora a resolução espacial dos dados. A partir de vídeos e principalmente imagens, diferentes dados da estrutura podem ser analisados via técnicas computacionais (SPENCER et al., 2019).

Além de imagens e vídeos convencionais, obtidas por câmeras, outros tipos de dados podem ser coletados da estrutura. Câmeras térmicas podem fornecer informações sobre a não uniformidade da estrutura e defeitos internos. Aparelhos de escaneamento a laser permitem identificar o alinhamento e nivelamento das peças. E inclinômetros possibilitam determinar a inclinação de superfícies (YAN et al., 2018). Dessa forma, o emprego de diferentes equipamentos possibilita o levantamento de variadas informações referentes à edificação, que ao serem analisadas emitirão o estado de conservação do elemento.

Todos os equipamentos relatados (câmeras convencionais e térmicas, aparelhos de escaneamento a laser e inclinômetros), e outros como GPS, sensores de obstáculos e fontes de luz, podem ser acoplados a veículos não 
tripulados (YAN, 2018), sejam eles aéreos, terrestres ou aquáticos. Nos tópicos subsequentes serão abordados cada um desses tipos de veículos e exemplos de aplicações na área de inspeção de estruturas.

\subsection{Veículos não tripulados aéreos}

\subsubsection{Conceito}

Os veículos aéreos não tripulados (VANTs) são popularmente conhecidos como drones. Na literatura são identificados pela sigla UAA, oriunda do termo em inglês unmanned aerial vehicle. Como a própria denominação indica, são veículos que não possuem pilotos a bordo. Seu controle, portanto, é feito remotamente por um operador em solo ou então por um conjunto de microprocessadores que o permitem funcionar autonomamente.

Os VANTs podem ser empregados em missões de observação e detecção, como: mapeamento de áreas, monitoramento de mudanças ambientais, identificação de desastres, exploração de recursos, entre outros. E sua grande vantagem nessas tarefas é o baixo custo e alta mobilidade quando comparado a veículos aéreos tripulados e ao sensoriamento remoto por satélites (LIU et al., 2014).

\subsubsection{Aplicações}

Dada sua grande mobilidade, rapidez e baixo custo, os VANTs tem um enorme potencial de uso na inspeção de edificações. Eles permitem que áreas de difícil acesso ou muito perigosas sejam alcançadas, como aquelas seriamente danificadas por catástrofes naturais. Os dados levantados em tempo real têm um benefício significativo para o planejamento de intervenção, limpeza e reabilitação da área acidentada (LIU et al., 2014).

Um caso que ilustra bem essa potencialidade foi o ocorrido no tsunami que atingiu o Japão em março de 2011 e destruiu quatro reatores da usina de Fukushima. Com a liberação de radiação, a inspeção humana do acidente se tornou extremamente problemática e perigosa. A solução encontrada foi o emprego de VANTs para fotografar a área destruída, fornecendo imagens dos danos. O modelo usado foi o T-Hawk, o mesmo empregado pelas forças militares americanas para identificação de bombas no Iraque (LIU et al., 2014).

A inspeção de pontes e viadutos é outra grande potencialidade do uso de VANTs. Usualmente, a avaliação dessas obras de arte é uma tarefa difícil, demandando muitas vezes veículos de inspeção de pontes (Fig. 1a). Embora forneçam acesso aos inspetores ao fundo das pontes, tais equipamentos são caros, atrapalham o trânsito e podem colocar os inspetores que os usam em risco. Por esse motivo, Tomiczek et al. (2018) desenvolveram um pequeno VANT com um sistema de navegação otimizado que permitia seu controle eficiente, mesmo com sinal insuficiente de GPS. Também foi equipado com um estabilizador de imagem e uma câmera de alta resolução, permitindo a obtenção de imagens do fundo da ponte (Fig. 1b e 1c).

Ainda nessa área, Karas (2015) relata o emprego de um hexacoptero equipado com uma pequena câmera térmica para avaliação das pontes Koberný e Ekanice e mais $10 \mathrm{~km}$ de rodovia na República Checa, em 2014. O trabalho foi uma parceria da empresa checa UpVision e da Direção de Estradas e Rodovias da República Checa (RMD), com o intuito de verificar problemas no isolamento, drenagem e irregularidades nas estruturas avaliadas. Com voos feitos ao fim da tarde, quando a temperatura da estrutura era maior, a equipe conseguiu avaliar a área e identificar satisfatoriamente as manifestações patológicas (Figura 2).

Quanto à área de transportes, as redes de estradas são uma importante parte da infraestrutura dos países e estão sujeitas a uma série de solicitações devido a carregamentos, condições ambientais e envelhecimento. Suas condições de rodagem são muito importantes para a manutenção dos sistemas de transportes. Por isso, devem ser inspecionadas e monitoradas rotineiramente, predominando atualmente a avaliação por pessoas treinadas que fazem extensivas observações de campo (ZHANG; ELAKSHER, 2012). Portanto, esse é mais um nicho que pode ser preenchido pela inspeção via VANTs.

Zhang e Elaksher (2012) empregaram um VANT para coleta de diversas imagens de uma rodovia não pavimentada americana. A partir do processamento das imagens adquiridas, conseguiram montar modelos tridimensionais da superfície da estrada (Figura 3), permitindo uma medição acurada do tamanho e dimensão de suas irregularidades. Com 


\section{CBPAT 2020

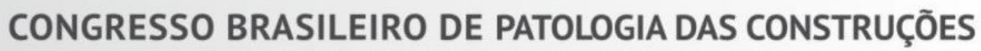 \\ DE 15 A 17 DE ABRIL | FORTALEZA - CE}

ISBN 978-65-86819-05-2

esta metodologia, as agências responsáveis pela conservação e manutenção das estradas podem ter acesso mais rápido, eficiente e segura às condições de acesso de suas vias.
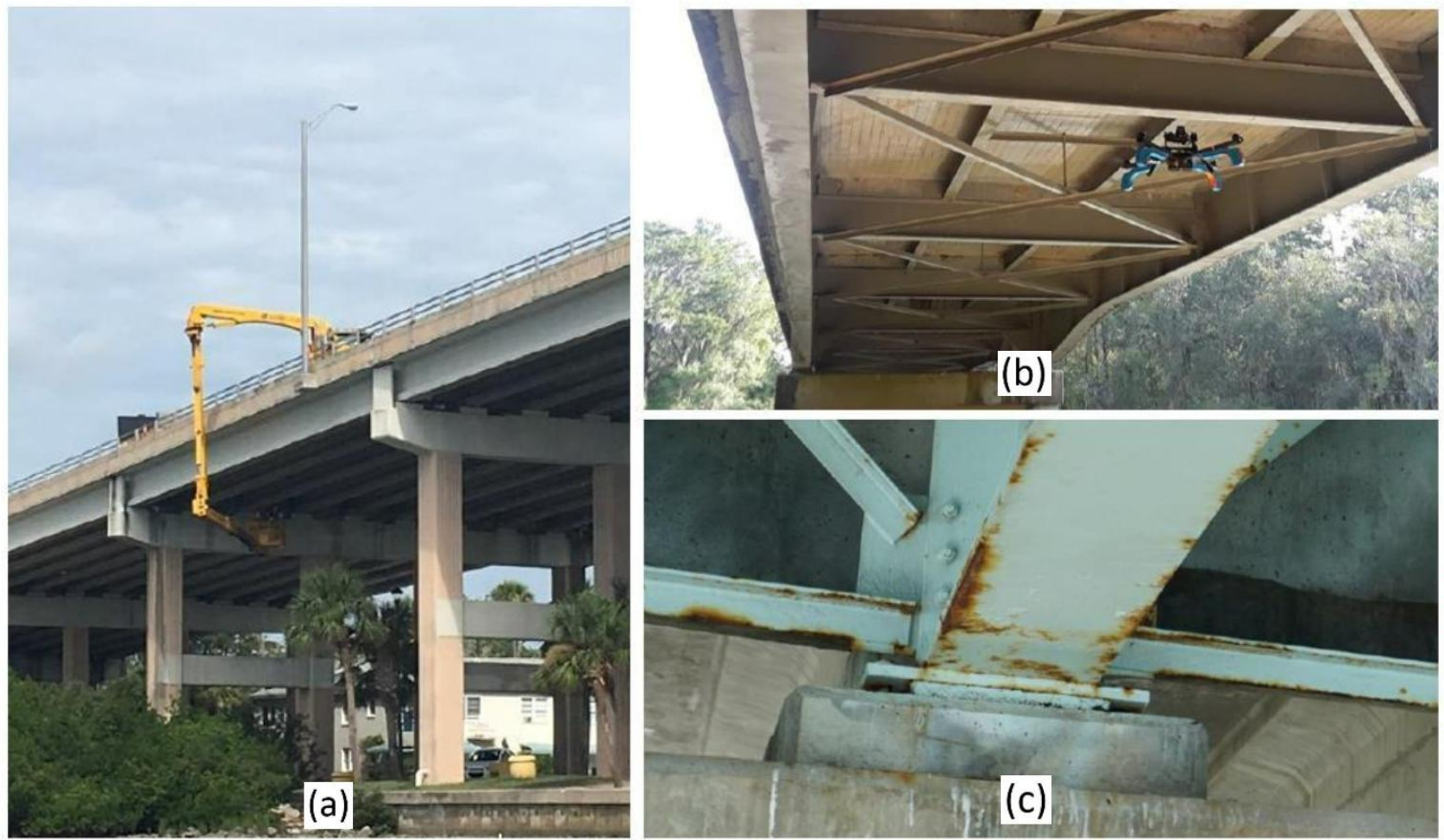

Figura 1: (a) Equipamento de vistoria de pontes usual; (b) VANT desenvolvido por Tomiczek et al. (2018) em serviço; (c) Imagem obtida pelo VANT evidenciando corrosão em peças do fundo de uma ponte.

FONTE: Tomiczek et al. (2018)
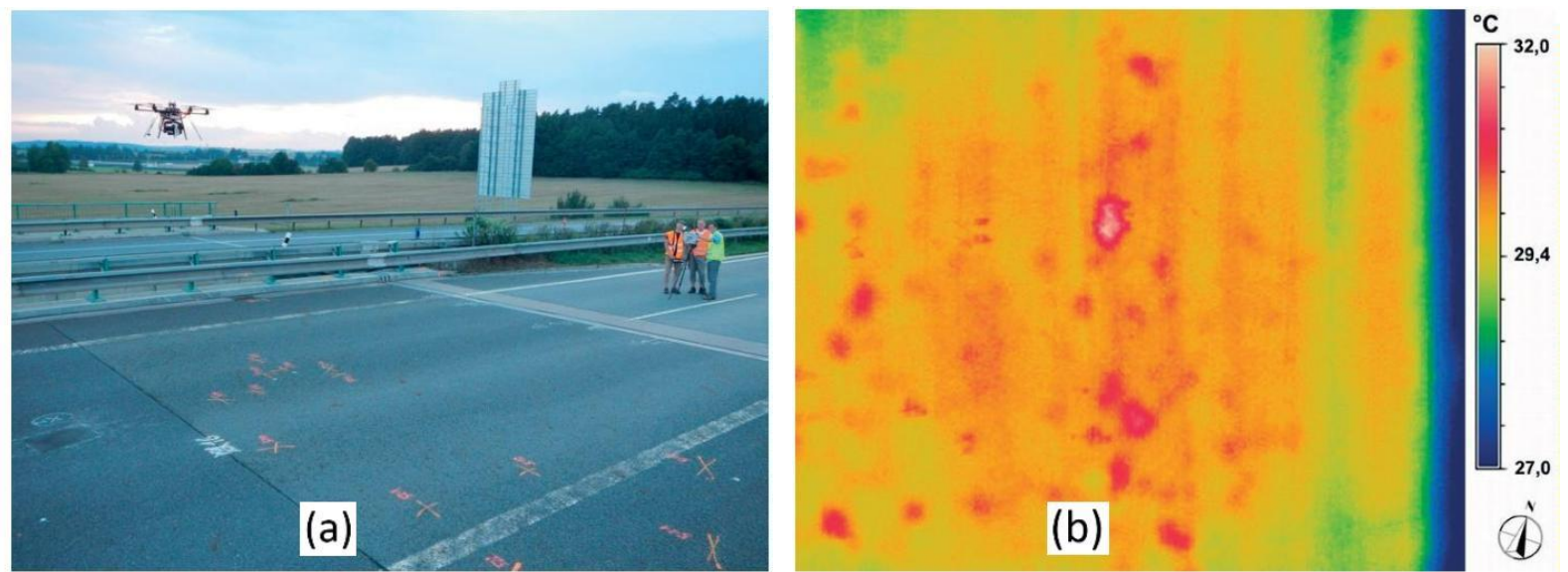

Figura 2: (a) Drone sobrevoando pontos críticos da ponte; (b) Imagem térmica mostrando protuberâncias e expansão de juntas entre isolamentos $8 \mathrm{~cm}$ abaixo da superfície.

FONTE: Karas (2015)

Outra grande potencialidade é a identificação de fissuras em estruturas de concreto, material este amplamente empregado na construção civil, como pontes, prédios e até mesmo usinas nucleares, dado seu bom custo-benefício e facilidade de moldar em diferentes formatos. No entanto, estruturas de concreto inevitavelmente sofrem fissuras devido a carregamentos e retração, sendo um indicador da saúde do elemento (KIM et al., 2017).

Nesse contexto, Kim et al. (2017) empregaram um VANT equipado com uma câmera, um sensor de deslocamento ultrassônico e um módulo WiFi (Figura 4a) para aquisição de informações referentes a fissuras em concreto (Figura 4b). 


\section{CBPAT 2020

Usando um processamento de imagens híbrido, a equipe conseguiu identificar com ótima precisão fissuras menores que $0,1 \mathrm{~mm}$.
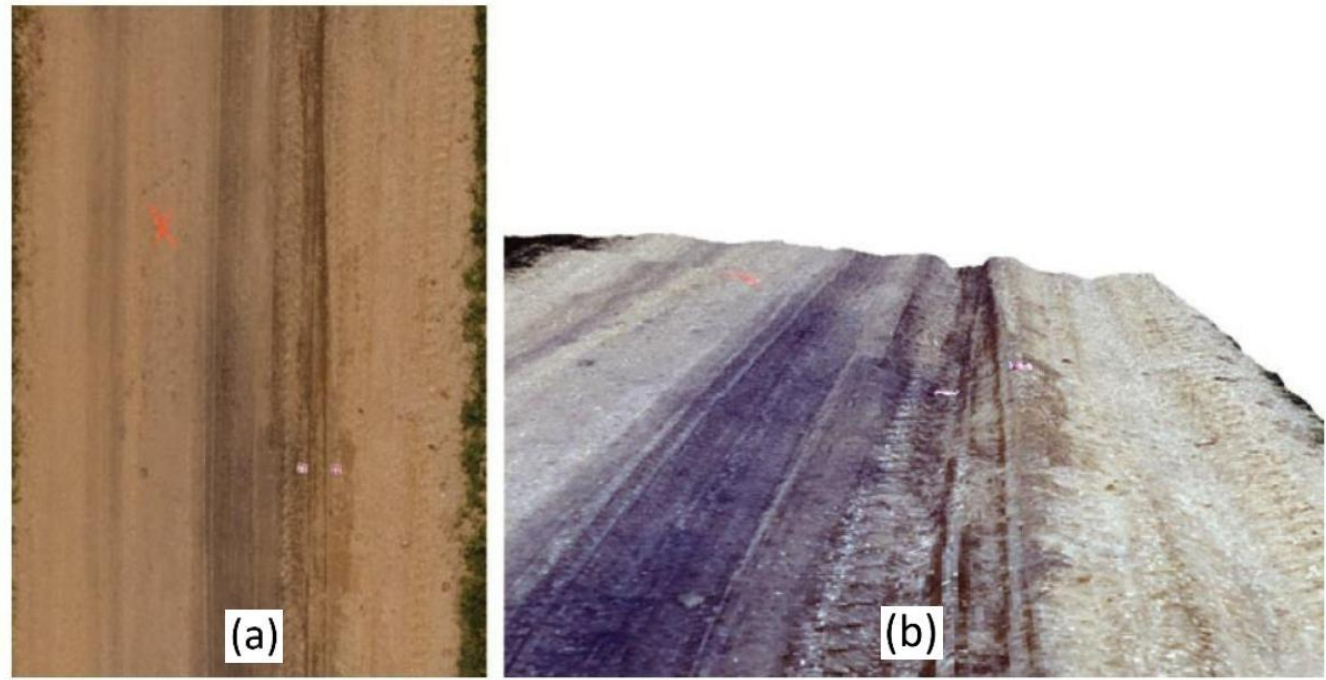

Figura 3: (a) Imagem aérea obtida pelo VANT; (b) Reconstituição 3D da superfície da estrada. FONTE: Zhang e Elaksher (2012)
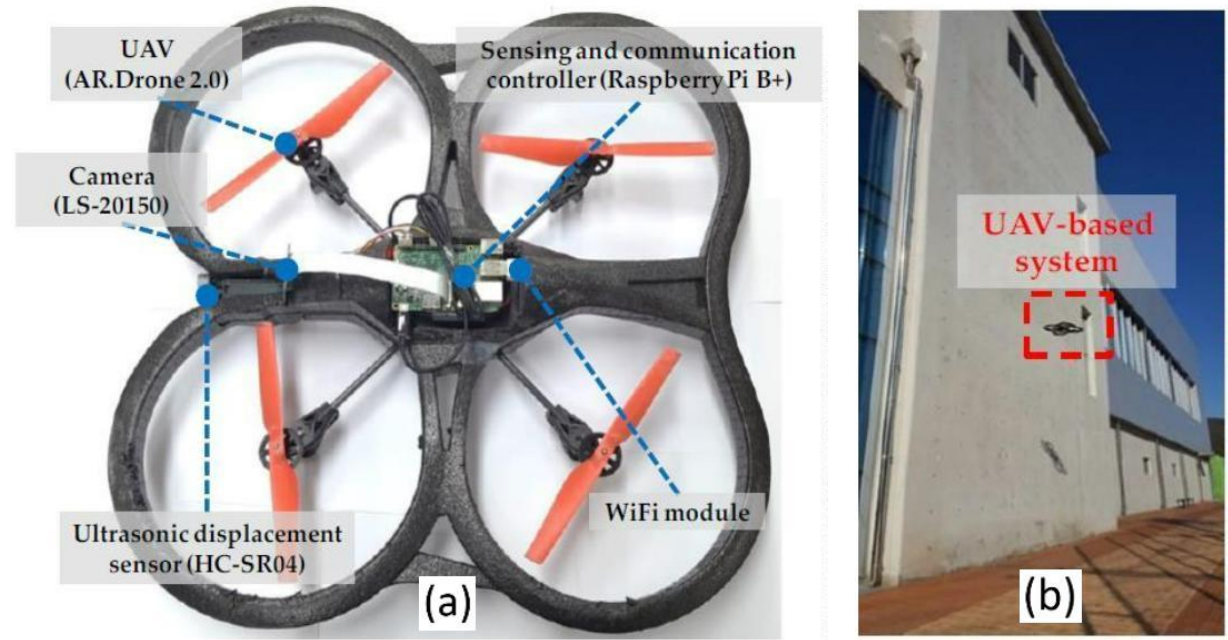

Figura 4: (a) Modelo e equipamentos acessórios do VANT para detecção de fissuras; (b) Validação do método de inspeção usando uma parede de concreto de um ginásio.

FONTE: Kim et al. (2017)

\subsection{Veículos terrestres não tripulados}

Enquanto os VANTs são mais pesquisados e empregados na construção civil, os veículos terrestres não tripulados (UGVs, do inglês unmanned ground vehicles) são utilizados de forma mais limitada, sobretudo em casos de catástrofes. Moud, Shojaei e Flood (2018), em uma revisão sobre a aplicação de veículos não tripulados terrestres e aquáticos, cita o emprego de UGVs no incidente do Word Trade Center, em 2001, e no furacão Katrina, em 2005.

Um exemplo da potencialidade no uso de UGVs em estruturas pós acidentes é o demonstrado por Torok, GolparvarFard, Kochersberger (2014). Eles empregaram um pequeno robô de solo equipado com uma câmera (Figura 5 a), que fez uma série de fotos de uma coluna de concreto altamente danificada, resultado de um ensaio de carregamento (Figura 5b). As imagens foram então processadas e deram origem a uma reconstituição 3D que caracterizou satisfatoriamente os danos da estrutura (Figura 5c). Assim, conseguiram demonstrar com o trabalho a eficiência do UGV e do processamento de imagens na inspeção de estruturas pós acidentes. 

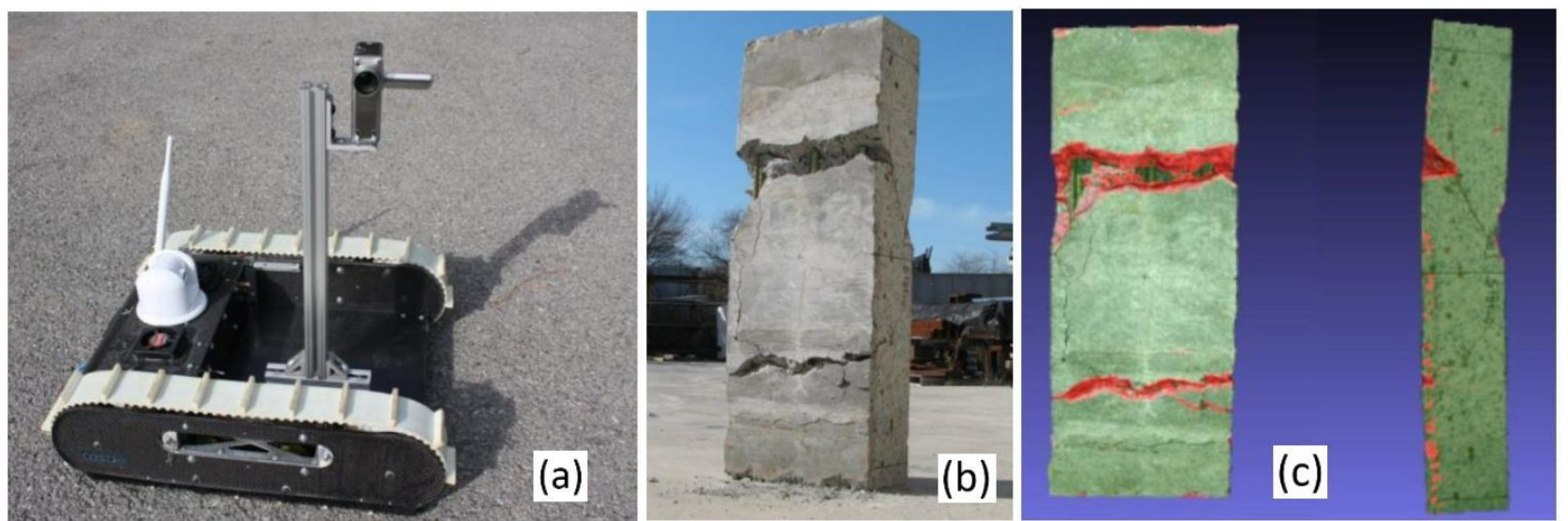

Figura 5: (a) UGV equipado com câmera digital e comunicação WiFi; (b) Coluna de concreto avaliada; (c) Reconstituição 3D após processamento das fotos obtidas pela UGV.

FONTE: Torok, Golparvar-Fard, Kochersberger (2014)

Yu, Jang e Han (2007) desenvolveram um UGV para detecção automática de fissuras em túneis. O robô foi equipado com sistemas de autofoco das fotos, redução de vibração, iluminação e codificadores para medir a velocidade e posição da unidade (figura 6a), permitindo um funcionamento automatizado da câmera. Com o processamento das imagens obtidas foi possível determinar o tipo de fissura, seu comprimento e profundidade (Figura 6b). Dada a ocorrência de alguns falsos positivos, a equipe optou por adotar um sistema semiautônomo de detecção, ou seja, havia a possibilidade de se descartar manualmente os pontos errôneos quando ocorressem.
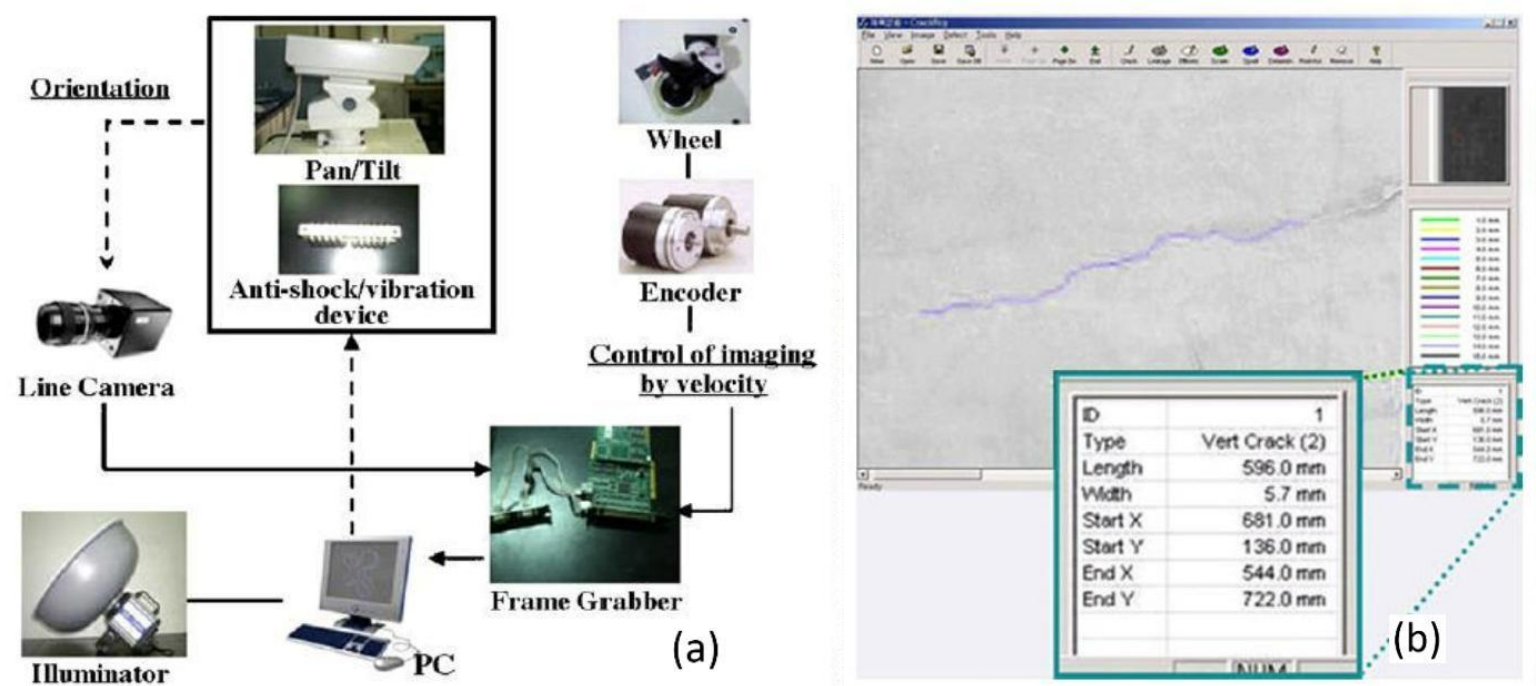

Figura 6: (a) Sistemas constituintes do UGV; (b) Identificação e medição de uma fissura.

FONTE: Yu, Jang e Han (2007)

Sutter et al. (2018) desenvolveram um robô semiautônomo para inspeção de pontes (Figura 7a), com o intuito de substituir a necessidade de os inspetores terem que escalar a estrutura para avaliação ou o emprego de equipamentos de vistoria de pontes. O dispositivo era composto por braços mecânicos onde robôs equipados com câmeras podiam se movimentar no sentido vertical e horizontal (Figura 7b). Todo o conjunto era comandado por uma pessoa dentro da cabine, sendo possível obter imagens das diferentes manifestações patológicas ocorrentes nas pontes, como as fissuras mostradas na Figura 7c.

\subsection{Veículos aquáticos não tripulados}

Existem três tipos de veículos aquáticos não tripulados: os UUV (do inglês unmanned underwater vehicles), os ROVs (remotely-operated vehicles) e os ASV (autonomous surface vehicles). Os UUV são veículos submersos que navegam sem conexão física à embarcação, ou seja, não são conectados a fios. Por analogia, podem ser considerados drones aquáticos. Os ROV são UUV conectados a embarcação por fios que fornecem energia e transmitem comandos dos 
operadores. E por fim, os ASV são veículos que trabalham na superfície da água ou semissubmersos. De forma geral, esses três tipos são denominados de UMVs (unmanned marine vehicles), pelo grande emprego em regiões litorâneas (MURPHY et al., 2011).
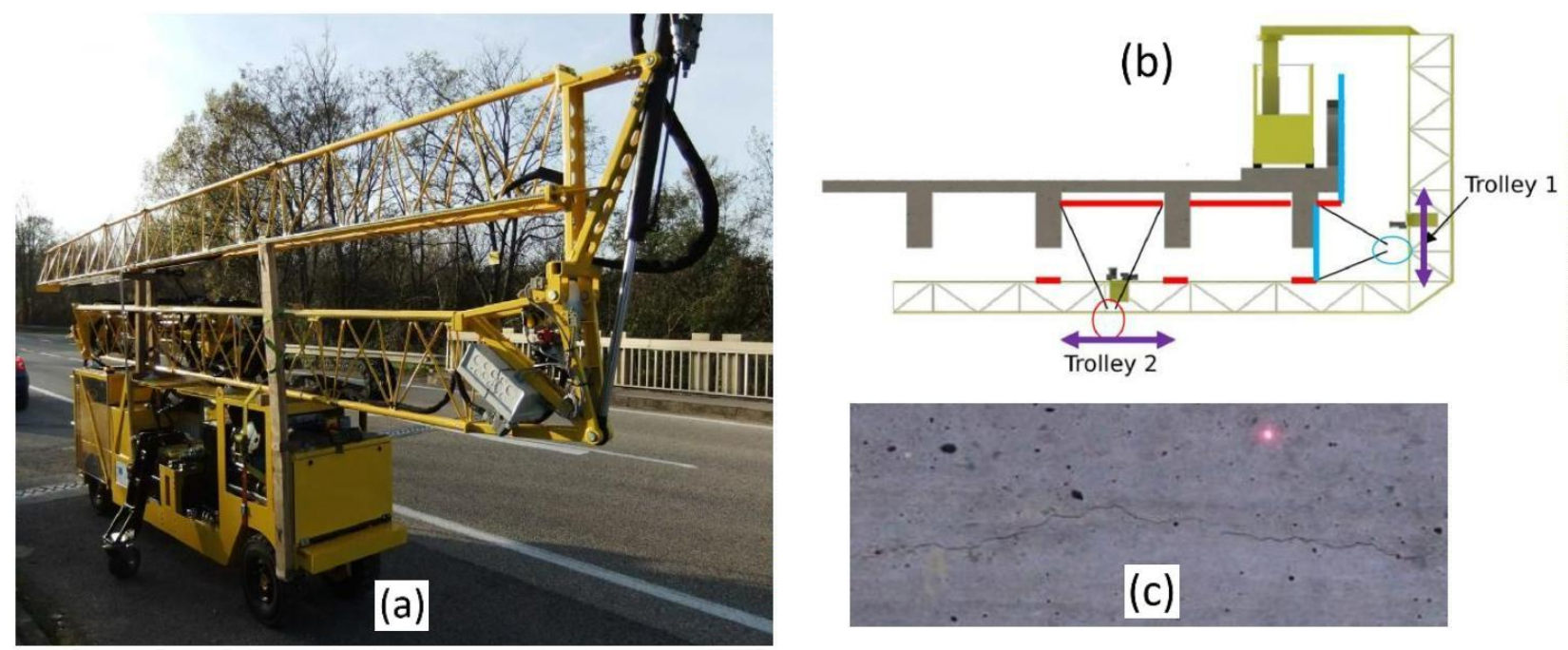

Figura 7: (a) Robô semiautônomo para inspeção de pontes; (b) Funcionamento do robô; (c) Fissura na superfície da ponte identificada pelo robô. FONTE: Sutter et al. (2018)

Assim como os UGVs, os veículos aquáticos não tripulados têm uso mais restrito na área de construção civil, sendo mais empregados na inspeção de estruturas após acidentes (MOUD; SHOJAEI; FLOOD, 2018).

Murphy et al. (2011) relata o uso dos três tipos de UMVs na inspeção da ponte Rollover Pass, no Texas, Estados Unidos, após a passagem do furacão Ike. Os veículos foram usados no mapeamento de escombros e inspeção da base da ponte. Para isso, eram equipados com câmeras fotográficas e sonares, fornecendo dados sobre as condições da estrutura.

A equipe ainda relata trabalhos similares efetuados na ponte Marco Island, danificada pelo furacão Wilma em 2005, (MURPHY et al., 2008) e na inspeção pelo FBI de uma ponte colapsada em Minneapolis em 2007, ambas as pontes nos Estados Unidos.

Embora o uso dos UMVs seja mais restrito, eles têm grande potencial na inspeção de estruturas com partes submersas e podem ser desenvolvidos veículos de baixo custo. Foi o que fizeram Manjunatha et al. (2018), que construíram um robô submerso com garras para inspeção da superfície externa de dutos. Ele era constituído por propulsores, duas garras para se prender aos dutos, um sensor giroscópico para manter a estabilidade na água e uma câmera de alta resolução para capturar imagens e vídeos em tempo real (Figura 8). O preço para construção do UMV ficou bem abaixo do ocorrente no mercado, demonstrando que o uso de robôs pode ser bem acessível e viável economicamente.

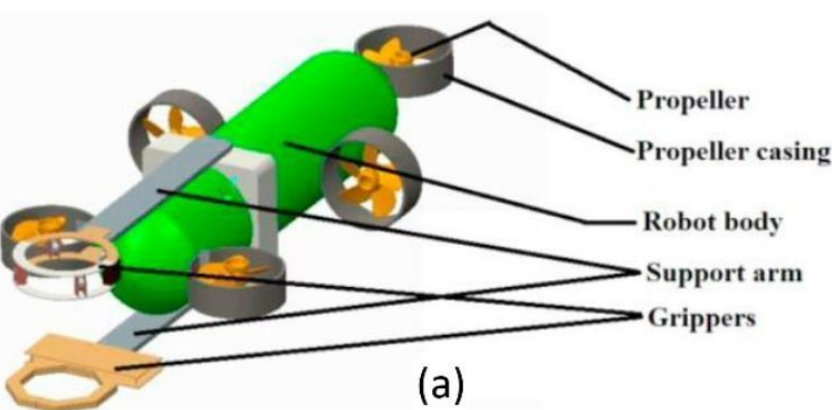

Figura 8: (a) Modelo em CAD do veículo aquático; (b) Veículo real. FONTE: Manjunatha et al. (2018)

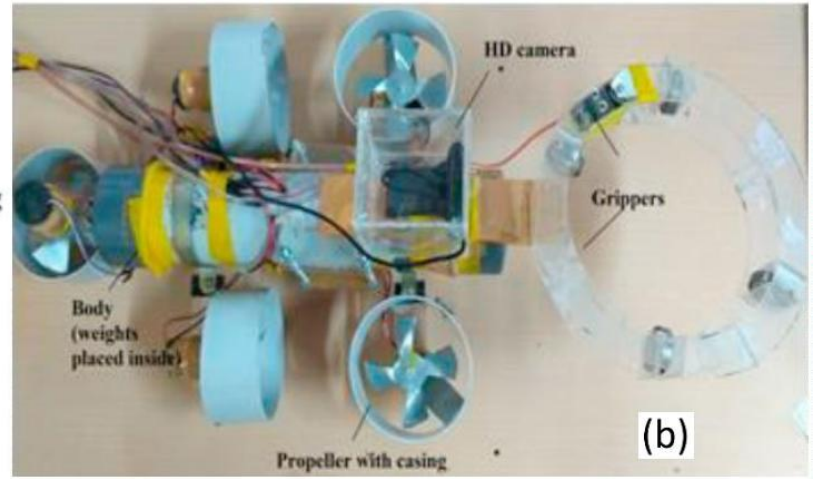

(b) 


\section{CONCLUSÕES}

Os veículos não tripulados são ferramentas de grande potencial para a área de inspeção e monitoramento de estruturas. Nesse sentido, tem-se observado um grande esforço para o desenvolvimento de veículos cada vez mais automatizados e do tratamento das imagens e outros dados obtidos por essas máquinas. O intuito é que se possa substituir a atividade humana, que possui limitações inerentes, como: subjetividade por parte dos inspetores na avaliação das manifestações patológicas; cansaço e baixo rendimento em inspeções de áreas muito extensas; dificuldade de acesso a algumas regiões, como fundos de pontes e fachadas muito altas; além de risco à vida em áreas na iminência de ruir ou que passaram por algum tipo de acidente natural, como terremotos, tsunamis e furações.

A grande vantagem do emprego de robôs está justamente em contornar todas essas limitações humanas citadas, tornando o monitoramento e inspeção de estruturas mais eficiente e seguro. E com o aprimoramento da tecnologia e popularização desse novo campo na Patologia das Construções, espera-se que as tarefas de inspeção se tornem algo mais comum, mais acessível e menos oneroso.

Cabe ressaltar que o emprego de UAVs, ou drones é atualmente bem mais disseminado que os outros tipos de veículos não tripulados. Isso se dá devido a sua grande mobilidade, podendo alcançar pontos críticos da estrutura com extrema facilidade, e seu baixo custo de operação. No entanto, os veículos terrestres e aquáticos também são muito promissores na inspeção de estruturas, tendendo a um crescimento de seu uso a medida em que mais pesquisas são realizadas e aprimoramentos dos equipamentos são feitos.

A modernização da Construção Civil é um dos pontos-chave para que se obtenha construções de maior qualidade, com menos erros e desperdícios, e de grande durabilidade. Deve-se, portanto, estar aberto para as novas tecnologias que surgem e integrá-las nas diferentes etapas de construção, incluindo as de monitoramento de suas condições ao longo de suas vidas úteis.

\section{REFERÊNCIAS}

HOSKERE, V. et al. Vision-based structural inspection using multiscale deep convolutional neural networks. In: Proceedings of the 3rd Huixian International Forum on Earthquake Engineering for Young Researchers; 2017 Aug $11-$ 12; Urbana, IL, USA; 2017.

KARAS, J. Mapping road and bridge damage in the Czech Republic: UASs for termal mapping. GIM International, 2015.

KIM, H. et al. Concrete Crack Identification Using a UAV Incorporating Hybrid Image Processing. Sensors, [s.1.], v. 17, n. 9, p.1-14, 7 set. 2017.

$\mathrm{KOCH}, \mathrm{C}$. et al. A review on computer vision based defect detection and condition assessment of concrete and asphalt civil infrastructure. Advanced Engineering Informatics, [s.1.], v. 29, n. 2, p.196-210, abr. 2015.

LICHTEnSteIn, N. B. Patologia das Construções. Boletim técnico n. 06. São Paulo - USP, 1986.

LIU, P. et al. A review of rotorcraft Unmanned Aerial Vehicle (UAV) developments and applications in civil engineering. Smart Structures And Systems, [s.1.], v. 13, n. 6, p.1065-1094, 25 jun. 2014.

MANJUNATHA, M. et al. A Low Cost Underwater Robot with Grippers for Visual Inspection of External Pipeline Surface. Procedia Computer Science, [s.1.], v. 133, p.108-115, 2018.

MOUD, H. I.; SHOJAEI, A.; FLOOD, I. Current and Future Applications of Unmanned Surface, Underwater, and Ground Vehicles in Construction. Construction Research Congress 2018, [s.1.], p.1-10, 29 mar. 2018.

MURPHY, R. R. et al. Cooperative use of unmanned sea surface and micro aerial vehicles at Hurricane Wilma. Journal Of Field Robotics, [s.1.], v. 25, n. 3, p.164-180, 2008. 
MURPHY, R. R. et al. Robot-Assisted Bridge Inspection. Journal Of Intelligent \& Robotic Systems, [s.1.], v. 64, n. 1, p.77-95, 18 jan. 2011.

SOUZA, V. C. M.; RIPPER, T. Patologia, recuperação e reforço de estruturas de concreto. São Paulo: Pini, 1998.

SPENCER, B. F.; HOSKERE, V.; NARAZAKI, Y. Advances in Computer Vision-Based Civil Infrastructure Inspection and Monitoring. Engineering, [s.1.], v. 5, n. 2, p.199-222, abr. 2019.

SUTTER, B. et al. A semi-autonomous mobile robot for bridge inspection. Automation In Construction, [s.1.], v. 91, p.111-119, jul. 2018.

TOMICZEK, A. P. et al. Small Unmanned Aerial Vehicle (sUAV) Inspections in GPS Denied Area beneath Bridges. Structures Congress 2018, [s.1.], p.205-2016, 17 abr. 2018.

TOROK, M. M.; GOLPARVAR-FARD, M.; KOCHERSBERGER, K. B. Image-Based Automated 3D Crack Detection for Post-disaster Building Assessment. Journal Of Computing In Civil Engineering, [s.1.], v. 28, n. 5, p.1-13, set. 2014.

YAN, R. et al. QuicaBot: Quality Inspection and Assessment Robot. IEEE Transactions On Automation Science And Engineering, [s.1.], v. 16, n. 2, p.506-517, abr. 2019.

YU, S, JANG, J,; HAN, C. Auto inspection system using a mobile robot for detecting concrete cracks in a tunnel. Automation In Construction, [s.1.], v. 16, n. 3, p.255-261, maio 2007.

ZHANG, C,; ELAKSHER, A. An Unmanned Aerial Vehicle-Based Imaging System for 3D Measurement of Unpaved Road Surface Distresses. Computer-aided Civil And Infrastructure Engineering, [s.1.], v. 27, n. 2, p.118-129, 21 jul. 2011. 